\title{
O SENSORIAMENTO REMOTO COMO FERRAMENTA PARA DETERMINAÇÃO DE BATIMETRIA DE BAIXIOS NA BAÍA DAS LARANJEIRAS, PARANAGUÁ - PR
}

\author{
Lilian Anne Krug ${ }^{1}$ e Mauricio Almeida Noernberg² \\ Recebido em 7 fevereiro, 2006 / Aceito em 2 maio, 2006 \\ Received on February 7, 2006 / Accepted on May 2, 2006
}

\begin{abstract}
Shallow waters represent potential risks to navigation inside estuaries. Therefore, they must be well described and data must be always updated. Traditional hydrographic methods have difficult to cover shallow areas due to them extension and low relief. An alternative is the use of satellite images for mapping the areas, with the advantage of low cost and quick results. In the north area of the Paranaguá Estuarine Complex the last bathymetric survey was in the 50's so a new bathymetric mapping of these areas, even without the nautical chart's accuracy, is useful for studies planning, environmental modeling and small boats navigation. This study had the objective of generate bathymetric map of shallow areas in the Laranjeiras bay through remote sensing. The application of this method is not difficult and could be very useful in regions were there is no bathymetric data or need to be updated. The map obtained is very realistic inside the limits of 0,36 to $4,5 \mathrm{~m}$ water depth and important considering that nearly $75 \%$ of the Paranaguá Estuarine Complex have depths less than $5 \mathrm{~m}$.
\end{abstract}

Keywords: shallow waters, remote sensing, bathymetry, Landsat.

RESUMO. Baixios representam riscos a navegação no interior dos estuários. Portanto, devem ser bem delineados e tais informações devem ser freqüentemente atualizadas. Métodos hidrográficos tradicionais têm dificuldade em cobrir áreas rasas devido sua extensão e relevo. Uma alternativa é 0 uso de imagens de satélites para mapear essas áreas, com as vantagens de baixo custo e rápido resultado. No setor norte do Complexo Estuarino de Paranaguá o último levantamento batimétrico foi realizado na década de 50 e um novo mapeamento destas áreas, mesmo sem a acurácia das cartas náuticas, é de utilidade para o planejamento de estudos, modelagem ambiental e navegação de pequenas embarcações. Este estudo teve o objetivo de avaliar o potencial de gerar mapa batimétrico das áreas rasas da Baía das Laranjeiras, através do sensoriamento remoto. A aplicação deste método é fácil e mostrou-se bastante útil em regiões onde não existem dados batimétricos ou estes precisam ser atualizados. 0 mapa obtido é bastante fiel dentro dos limites batimétricos que se pretende, entre 0,36 e 4,5 m de profundidade, principalmente considerando que $75 \%$ do complexo estuarino possui profundidade inferior a $5 \mathrm{~m}$.

Palavras-chave: baixios, sensoriamento remoto, batimetria, Landsat.

\footnotetext{
${ }^{1}$ Instituto Nacional de Pesquisas Espaciais, Divisão de Sensoriamento Remoto, Av. dos Astronautas, 1758, 12.227-010 São José dos Campos, SP, Brasil. Tel: (12) 3945-6441; Fax: (12) 3945-6449 - E-mail: licakrug@dsr.inpe.br

${ }^{2}$ Centro de Estudos do Mar - UFPR, Av. Beira-mar, s/nº, Cx. Postal 50.002, 83255-000. Pontal do Paraná, PR, Brasil. Tel: (41) 3455-1333; Fax: (41) 3455-2586

-E-mail: m.noernberg@ufpr.br
} 


\section{INTRODUÇÃo}

Baixios (ou bancos arenosos) são elevações do fundo marinho, lagunar ou estuarino, que estão em constante alteração morfológica devido a hidrodinâmica. Suas formas de fundo ou bancos podem estar submersos ou semi-submersos, em baixas profundidades, sendo formados por material inconsolidado, geralmente arenoso (Suguio, 1992). 0 fato de os baixios terem formas de fundo dinâmicas e representarem áreas de perigo às embarcações torna necessário o levantamento batimétrico constante para maior segurança à navegação.

0 Complexo Estuarino de Paranaguá (CEP) está localizado no litoral sul brasileiro ( $48^{\circ} 22^{\prime} \mathrm{W}, 25^{\circ} 28^{\prime} \mathrm{S}$ ) e é um sistema ambiental de extrema importância, de navegação intensa, seja por atividades ligadas à pesca artesanal, à recreação e ao turismo, bem como comercial, sendo esta última ligada principalmente à atividade portuária.

0 CEP é separado em dois eixos principais (Fig. 1): no eixo L-0, estão as baías de Paranaguá e Antonina com seus portos homônimos, sendo o porto de Paranaguá o maior exportador de grãos da América Latina. A importância de manutenção do calado do canal de acesso aos portos, faz com que neste eixo o mapeamento batimétrico esteja sempre atualizado. Por outro lado, no eixo N-S, que compreende a baía das Laranjeiras, o sub-estuário de Guaraqueçaba e 0 canal de acesso interno a baía dos Pinheiros, 0 último levantamento batimétrico data de 1945 e considera apenas a parte mais externa da baía das Laranjeiras. Embora não exista tráfego de embarcações de grande calado, a presença de muitas vilas e pontos de pesca neste eixo do CEP faz com que pequenas embarcações estejam sempre em circulação.

Levantamentos batimétricos realizados com o auxílio de ecobatímetro são de alto custo e demorados, pois necessitam de pessoal, equipamentos, embarcações e muito trabalho de campo. Além disso, na maioria das vezes não compreendem regiões com pouca profundidade devido à dificuldade de navegação. Uma alternativa é o uso do sensoriamento remoto para o mapeamento de extensas áreas rasas (Tedesco \& Centeno, 2003; Ryu et al., 2004). Imagens de satélites provêm, a baixos custos, mapas batimétricos de áreas que são difíceis de mapear por meios hidrográficos tradicionais devido à sua extensão ou seu complexo relevo submerso.

Esse tipo de metodologia pôde ser aplicada no CEP devido suas extensas áreas rasas. Segundo Noernberg (2001), 75\% da superfície líquida do CEP possui profundidade inferior a $5 \mathrm{~m}$.

0 presente estudo tem como objetivo avaliar o potencial de uso de imagens Landsat na geração de mapa batimétrico em áreas rasas.

\section{METODOLOGIA \\ Revisão teórica}

A literatura apresenta diversos modelos que visam extrair informações batimétricas da coluna d'água a partir da resposta recebida pelo sensor orbital. Além de métodos que utilizam valores de radiância, há os que trabalham diretamente com os valores digitais da imagem, sem necessidade de transformação dos mesmos em radiância (Tedesco \& Centeno, 2003).

0 modelo mais simples de extração de profundidade a partir de imagens de satélite trabalha com uma única banda, embora seja possível adaptar a equação abaixo descrita ao uso de mais de uma banda (Lyzenga, 1978; Nordman et al., 1990).

Segundo Philpot (1989), de forma geral:

$$
L_{d}=L_{b} \exp (-g z)+L_{w}^{\prime}
$$

onde $L_{d}$ é a radiância observada pelo sensor; $g 0$ coeficiente de atenuação efetiva da água; $z$ a profundidade da coluna d'água; $L_{b}$ é a reflectância do fundo e $L_{w}$ a radiância observada sobre água oticamente profunda.

Ao utilizar este modelo, assume-se que as propriedades ópticas da água são verticalmente homogêneas, bem como, consideram-se constantes sobre a cena a iluminação, a transmissão atmosférica, 0 trajeto da radiância, o estado do mar, a reflectância da água, a reflectância do fundo e a atenuação efetiva da água. Assim, presumindo-se que os parâmetros $L_{b}, L_{w}$ e $g$ são constantes sobre a cena, e sabendo que os valores de $L_{d}$ para uma ou mais profundidades conhecidas, pode-se encontrar os valores de $L_{b}$ e $g$. Sempre que $L_{w}$ é tomado como constante, o procedimento usual é "linearizar" a equação (1), definindo, desta forma, uma nova variável $(X)$, a qual varia linearmente com a profundidade:

$$
X=\ln \left(L_{d}-L_{w}\right)=\ln \left(L_{b}\right)-g z .
$$

Um outro método desenvolvido para delinear feições na água e realçá-las em uma imagem de satélite multiespectral é o Índice de Diferença Normalizada da Água (Normalized Difference Water Index - NDWI). 0 NDWI faz uso do sinal refletido nas regiões do infravermelho próximo e do verde visível para realçar a presença de tais feições enquanto elimina a influência do solo e vegetação terrestre (McFeeters, 1996). 0 índice é calculado por:

$$
\frac{D N_{2}-D N_{4}}{D N_{2}+D N_{4}}
$$

onde $\mathrm{DN}_{2}$ é o valor digital do pixel na banda 2 do Landsat, correspondente da faixa verde do espectro visível e $D N_{4}$, valor digital na banda 4, região do infravermelho próximo. 


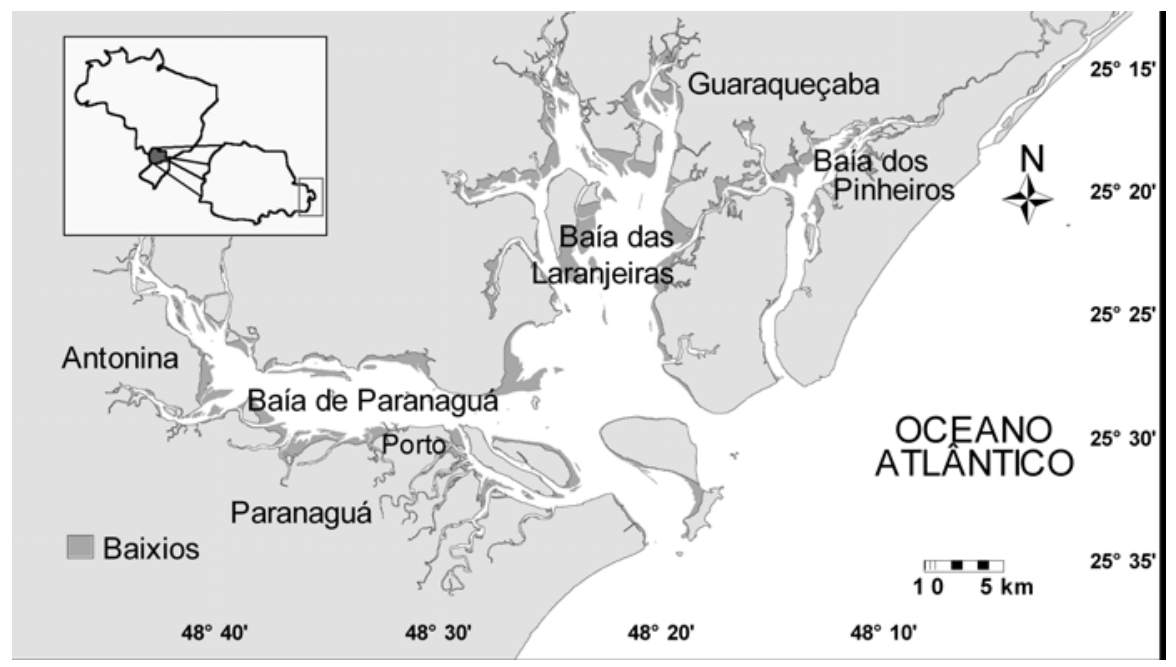

Figura 1 - 0 Complexo Estuarino de Paranaguá - PR.

De acordo com McFeeters (1996), a seleção destas bandas tem a intenção de: (1) maximizar a reflectância típica de feições na água nos comprimentos de onda relativos ao canal verde; (2) minimizar a baixa reflectância no infravermelho próximo de feições na água; e (3) utilizar a alta reflectância no infravermelho próximo por feições de vegetação terrestre e solo.

\section{Medidas em laboratório}

Para obter a equação que fornece a relação entre o valor digital da imagem e a profundidade da coluna d'água foram utilizados dados batimétricos levantados pela Diretoria de Hidrografia e Navegação da Marinha do Brasil (DHN) em frente ao porto de Paranaguá. Os valores de profundidade e do valor digital de cada ponto foram correlacionados em diferentes composições de bandas da imagem e em diferentes limites de profundidade, para selecionar entre elas a que possui o melhor valor de correlação (Krug \& Noernberg, 2005).

A imagem utilizada foi adquirida pelo sensor ETM+ do satélite Landsat 7 em 26 de setembro de 1999, devidamente corrigida de distorções radiométricas, geométricas e atmosféricas, esta última corrigida pelo método de regressão linear descrito por Mather (1987).

Os dados batimétricos utilizados foram levantados pela DHN em agosto de 1998 e tratados para eliminar o efeito da maré na profundidade. Posteriormente, foi adicionada a altura da maré no momento da aquisição da imagem, permitindo a comparação dos valores digitais da imagem com a profundidade real no momento da aquisiç̧ão desta.

\section{RESULTADOS E DISCUSSÃO}

Entre as composições avaliadas o NDWI apresentou o melhor coeficiente de determinação em um maior intervalo de profundidades. 0 NDWI apresentou uma tendência logarítmica em relação à variação da profundidade (Fig. 2).

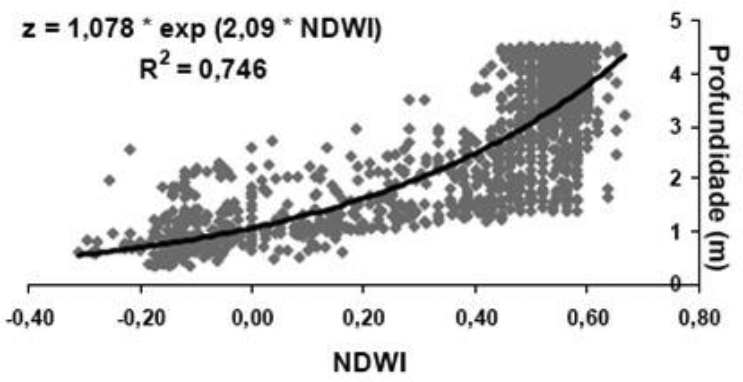

Figura 2 - Comportamento dos valores digitais do NDWI em relação à variação de profundidade.

0 mapa batimétrico (Fig. 3) gerado a partir do Índice de Diferença Normalizada da Água, para o intervalo de 0,36 a 4,5 metros de profundidade, com correlação de Pearson $(r)$ de 0,814 , coeficiente de determinação $\left(R^{2}\right)$ de 0,746 e erro médio inferior a 0,65 m, foi obtido a partir da seguinte equação:

$$
Z=1,078 * \exp \left(2,09 *\left(\frac{D N_{2}-D N_{4}}{D N_{2}+D N_{4}}\right)\right) .
$$

Para analisar o padrão dos erros da resposta do modelo de acordo com o aumento da profundidade, foram plotados os valores da profundidade real subtraídos dos valores da profundidade estimada (Fig. 4). É possível notar as diferentes influências na resposta obtida pelo sensor. A maior variação do erro, entre $1 \mathrm{e}$ 3,5 metros de profundidade, atribuí-se à influência na reflectância, 


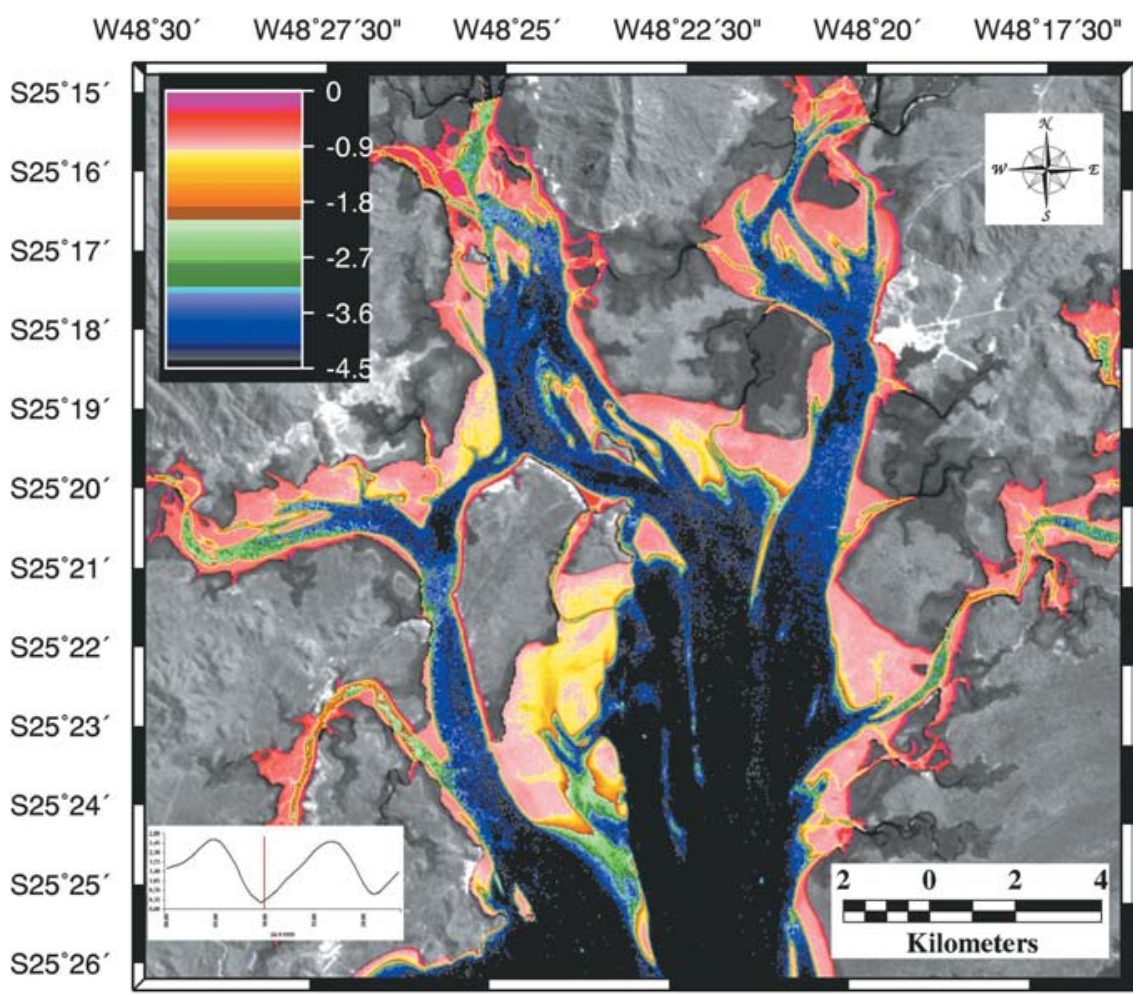

Figura 3 - Batimetria estimada para 0 setor norte do Complexo Estuarino de Paranaguá a partir do Índice de Diferença Normalizada da Água - NDWI. As profundidades estão em metros. 0 gráfico mostra a altura da maré na hora da aquisição da imagem pelo satélite Landsat 7 - ETM+.

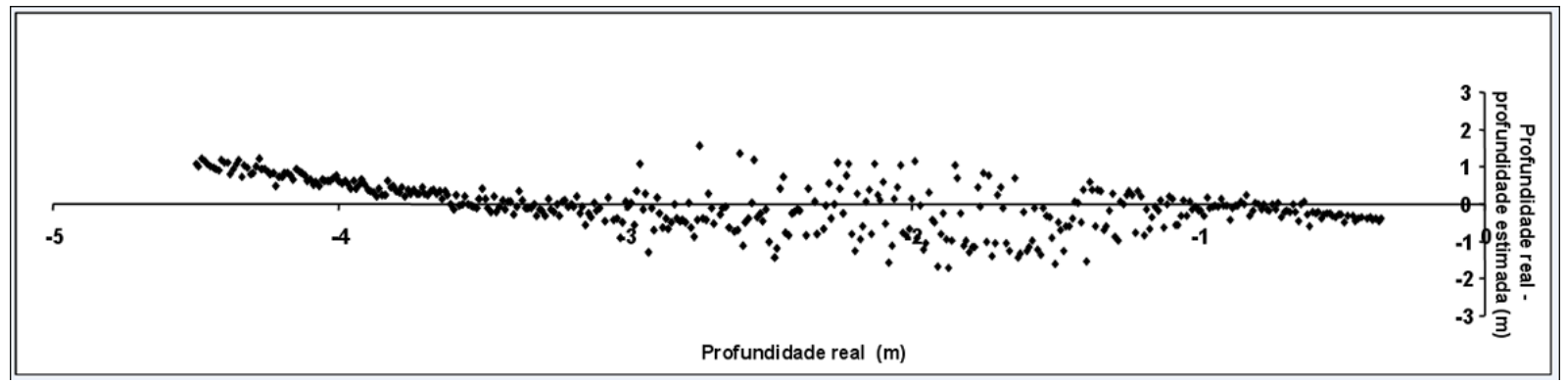

Figura 4 - Padrão dos erros para o Índice de Diferença Normalizada da Água com intervalos de profundidade de 0,36 a 4,5 m.

tanto do fundo quanto da coluna d'água e seus constituintes opticamente ativos (material particulado em suspensão).

0 valor de correlação de Pearson entre os valores de profundidade estimados pelo modelo e as profundidades verdadeiras foi de 0,83. Green et al. (2000) encontraram valores entre 0,52 e 0,91 em quatro diferentes métodos testados em ambientes de recifes de coral, onde a água possui menor turbidez. Isto pode ser considerado um bom resultado dado às características ópticas das águas estuarinas.

\section{CONCLUSÕES}

Técnicas de sensoriamento remoto têm sido empregadas por muitos investigadores para obter informações sobre os ambientes marinhos e costeiros. 0 conhecimento e o entendimento das heterogeneidades temporais e espaciais de estuários são de suma importância para o seu manejo. A questão mais difícil para extração de informações a respeito da profundidade e outros parâmetros da coluna d'água está em distinguir as diferentes variações cau- 
sadas por alterações na profundidade da água, tipo de fundo ou por espalhamento e absorção do comprimento de onda na coluna d'água.

Nota-se na Figura 3 a interferência do material em suspensão na reflectância em locais de correntes fortes, como os canais de maré, esta causada por processos de ressuspensão de sedimentos de fundo, limitando os intervalos de profundidade possíveis de serem determinados. 0 ideal é que essas influências possam ser identificadas e quantificadas e desta forma, consideradas no algoritmo, visto que as condições ambientais podem ter uma maior influência na acurácia da técnica.

0 presente estudo foi conduzido com a intenção de avaliar a aplicabilidade de imagem Landsat na extração de informações batimétricas de baixios no setor norte do Complexo Estuarino de Paranaguá. Os resultados obtidos são fiéis dentro do limite de profundidade aos quais se pretendem, podendo prover uma contribuição valorosa no conhecimento do relevo subaquático da região.

A carta náutica 1820, a qual compreende a baía das Laranjeiras mostra valores de profundidade medidos décadas atrás. 0 mapa batimétrico construído a partir da imagem de satélite pode complementar a carta náutica, pois soma a ela dados de regiões que não são possíveis de sondar com ecobatímetros. Mesmo sem a acurácia similar, o mapa é de utilidade tanto para o planejamento de estudos e para a modelagem ambiental, quanto para a segurança da navegação de embarcações de pequeno porte.

Para estudos futuros sugere-se a comparação entre os mapas batimétricos obtidos por sensores remotos orbitais e as informações batimétricas obtidas por métodos tradicionais. Esta comparação além de quantificar a variação batimétrica no tempo possibilita, também, inferências a respeito da dinâmica sedimentar da região.

Os sensores TM e ETM+ da série de satélites Landsat registram informações da superfície terrestre desde a década de 80 . 0 método descrito aqui pode ser aplicado na análise da evolução morfológica de ambientes onde alterações são significativas em escalas de poucos anos a décadas a partir destas imagens.

\section{REFERÊNCIAS}

GREEN EP, MUMBY PJ, EDWARDS AJ \& CLARK CD. 2000. Remote Sensing Handbook for Tropical Coastal Management. Coastal Management Sourcebooks 3. Paris: UNESCO. 316 pp.

KRUG LA \& NOERNBERG MA. 2005. Extração de batimetria por sensoriamento remoto de áreas rasas dos sistemas estuarinos do Estado do Paraná - Brasil. Anais do XII Simpósio Brasileiro de Sensoriamento Remoto. Goiânia, GO. CD-ROM.

LYZENGA DR. 1978. Passive Remote Sensing Techniques for Mapping Water Depth and Bottom Features. Applied Optics, 17(3): 379-383.

MATHER PM. 1987. Computer processing of remotely-sensed images: an introduction. Nova York: Willey. 352 pp.

McFEETERS SK. 1996. The use of the Normalized Difference Water Index (NDWI) in the delineation of open water features. Int. Journal of Remote Sensing, 17(7): 1425-1432.

NOERNBERG MA. 2001. Processos morfodinâmicos no complexo estuarino de Paranaguá - Paraná - Brasil: um estudo a partir de dados in situ e Landsat-TM. Tese de Doutorado, Universidade Federal do Paraná. Curitiba, PR. 179 pp

NORDMAN ME, WOOD L, MICHALEK JL \& CHRISTY JJ. 1990. Water depth extraction from Landsat- 5 imagery. Proceedings of the Twentythird International Symposium on Remote Sensing of Environment. p. $1129-1139$

PHILPOT WD. 1989. Bathymetric Mapping with Passive Multispectral Imagery. Applied Optics, 28(8): 1569-1578.

RYU J, NA Y, WON J \& DOERFFER R. 2004. A critical grain size for Landsat ETM+ investigations into intertidal sediments: a case study of the Gomso tidal flats, Korea. Estuarine, Coastal and Shelf Science, 60: 491-502.

SUGUIO K. 1992. Dicionário de Geologia Marinha. Ed. T.A. Queiroz, São Paulo. $171 \mathrm{pp}$.

TEDESCO A \& CENTENO JAS. 2003. Utilização de imagens Ikonos para estimativa de profundidades. Anais do III Colóquio Brasileiro de Ciências Geodésicas. Curitiba, PR. Disponível em: <http://www.milenio.com.br/jed/inicio>. Acesso em: 02 jun. 2003. $14 \mathrm{pp}$.

\section{NOTAS SOBRE OS AUTORES}

Lilian Anne Krug. Oceanógrafa formada pela Universidade Federal do Paraná - UFPR 2004. Mestranda de Sensoriamento Remoto no Instituto Nacional de Pesquisas Espaciais - INPE.

Mauricio Almeida Noernberg. Oceanólogo pela Fundação Universidade Rio Grande - FURG 1988. Mestrado em Sensoriamento Remoto pelo Instituto Nacional de Pesquisas Espaciais - INPE. Doutor em Geologia Ambiental pela Universidade Federal do Paraná, 2001. Professor Adjunto do Centro de Estudos do Mar da UFPR e responsável pelo Laboratório de Oceanografia Costeira e Geoprocessamento. Tem atuado em estudos de processos costeiros através de sensoriamento remoto e medidas in situ. 